\title{
Digitization of partitions and tessellations
}

\author{
Jean Serra ${ }^{1}$ and B Ravi Kiran ${ }^{2}$ \\ 1 Université Paris-Est, A3SI-ESIEE LIGM \\ jean.serra@esiee.fr \\ 2 Center de robotique, MINES ParisTech, PSL-Research University, \\ ravi.kiran@mines-paritech.fr
}

\begin{abstract}
We study hierarchies of partitions in a topological space where the interiors of the classes and their frontiers are simultaneously represented. In both continuous and discrete spaces our approach rests on tessellations whose classes are $\mathcal{R}$-open sets. In the discrete case, the passage from partitions to tessellations is expressed by the Alexandrov topology and yields double resolutions. A new topology is proposed to remove the ambiguities of the diagonal configurations. It leads to the triangular grid in $\mathbb{Z}^{2}$ and the centered cubic grid in $\mathbb{Z}^{3}$, which are the only translation invariant grids which preserve connectivity and permit the use of saliency functions.
\end{abstract}

Keywords: Tessellations, Regular sets, Hierarchies, Khalimsky topology, Simplicial complexes, 3 -D grids.

\section{Introduction}

When a set is partitioned, the frontiers between classes are not explicitly addressed, while the operations on these frontiers might not trivial. Does the frontier between two classes belong to one of them, to both, or to none? How does one combine set interiors and frontiers in a unique representation? This is the focus of the current study.
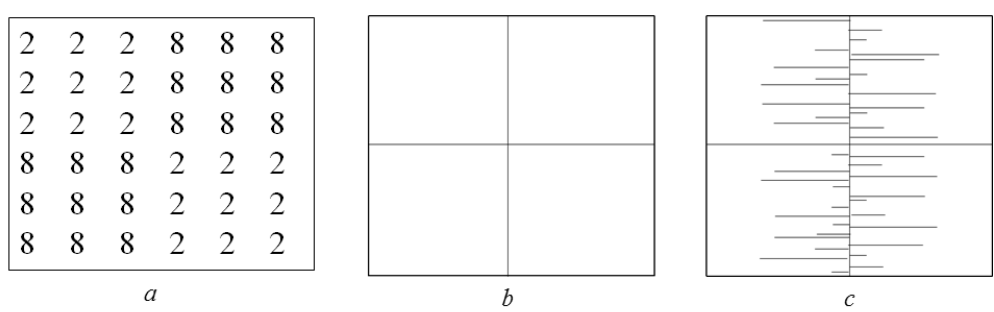

Fig. 1. a) In a hierarchy, must we firstly connect the "2"s, or the "8"s? Do the frontiers in Figure c) play the same role as in Figure b)?

When one goes up in a hierarchy of partitions, the frontier elements which disappear are only those separating classes, e.g. the two medians in Fig.1b, and not the barbs of Fig. 1c. How does one differentiate between these two cases? An answer is given by the notion of tessellation (section $3)$.

Preserving connectivity within classes may also be troublesome. How does one merge the squares of Fig. 1a? Must we firstly link the "2"s, or rather the "8"s ? In 2D, the classical digital approach focuses on the boundaries, which are usually supposed to be Jordan curves [13,22]. One could also constructs "well composed set", whose boundaries have no diagonal configurations [14].

In this study, our intuition is based on the fact that the classes themselves, and not their boundaries, would provide a more convenient input to the present problem. We will see that Jordan curves are totally useless in what follows, though the particular structure of the classes $(\mathcal{R}$-open sets) is crucial.

Image processing rests either on Euclidean background, or on the digital one, but the problem of the frontiers in a partition is set upstream this distinction, and its solution will apply to both 
continuous and discrete models. In the second case Khalimsky topology is a convenient starting point, for it identifies $\mathbb{Z}^{n}$ with Euclidean $n$-cubes $[1,17]$. It involves simplicial complexes which lead to double resolution techniques $[3,4,9]$ (section 5). But it does not preserve connectivity (see Fig. 1a), unlike the topological variant developed in section 6 .

\section{Lattice of the regular open sets (reminder)}

Let $E$ be a set equipped with a (non necessarily separated) topology, and $\mathcal{G}$ be the class of its open sets. $\mathcal{G}$ is a complete lattice, but not complemented. Now, the notion of a complement is essential, and this orients us toward the family $\mathcal{R}=\mathcal{R}(E)$ of the regular open sets of $E$. An open set $B$ is said to be regular, or $\mathcal{R}$-open, when it does not change when one takes its adherence $\bar{B}$, and then one takes the interior of the latter, i.e. $B=(\bar{B})^{\circ}$. It is the case of Fig.1b for example, but not of Fig.1c. ${ }^{3}$. The main result about $\mathcal{R}$ is the following theorem $[6,7,21]$ :

Theorem 1. $\mathcal{R}$ is a complete lattice for the inclusion ordering, where the supremum and the infimum are given by

$$
\vee B_{i}=\left(\overline{\cup B_{i}}\right)^{\circ} ; \wedge B_{i}=\left(\cap B_{i}\right)^{\circ} .
$$

Lattice $\mathcal{R}$ is completely distributive and with unique complement

$$
\operatorname{compl} B=(\complement B)^{\circ} .
$$

Here the symbol $\complement$ designates the set complement operator. For example, in the two Fig. 4a and $\mathrm{b}$, the complement of each class is the supremum, in $\mathcal{R}$, of the three other ones.

\section{Tessellations}

A "tessellation" is a partition of a topological space where both interiors and their boundaries are classes [25]:

Definition 1. We define a tessellation $\tau$ of a topological space $E$ as any family $\left\{B_{i}, i \in I\right\}$ of disjoint open sets called "classes":

$$
\tau=\left\{B_{i}, i \in I\right\} \text { with } i \neq j \Rightarrow B_{i} \cap B_{j}=\varnothing
$$

such that the union of all $B_{i}$ and of all boundaries $\operatorname{Fr}\left(B_{i}, B_{j}\right)=\bar{B}_{i} \cap \bar{B}_{j}$ covers the space E:

$$
E=\cup\left\{B_{i}, i \in I\right\} \cup\left\{\bar{B}_{i} \cap \bar{B}_{j}, i, j \in E, i \neq j\right\},
$$

We will designate by $N$ ( $N$ for "net") the set $\cup\left\{\bar{B}_{i} \cap \bar{B}_{j}, i, j \in E, i \neq j\right\}$ of all boundaries between classes.

\subsection{Tessellations and $\mathcal{R}$ open sets}

In what follows, $S=(\bar{B})^{\circ}$ designates the $\mathcal{R}$-open transform of the open set $B \in \mathcal{G}(E)$. The operation $B \rightarrow S=(\bar{B})^{\circ}$ is an algebraic closing on the set $\mathcal{G}$ of the open sets of $E$, and the image of $\mathcal{G}$ is $\mathcal{R}$. For example, if we take Fig. 1c for $B$, we obtain Fig. 1b for transform $S$. This closing means that $S$ is the smallest $\mathcal{R}$-open set that contains $B$. Indeed, if another $\mathcal{R}$-open $S^{\prime}$ contains $B$, then $S^{\prime}=\left(\overline{S^{\prime}}\right)^{\circ} \supseteq(\bar{B})^{\circ}=S$. Note that

$$
S=(\bar{B})^{\circ} \quad \Rightarrow \quad S \supseteq B \quad \text { and } \quad \bar{S}=\bar{B} . \quad B \in \mathcal{G}
$$

${ }^{3}$ In mathematical morphology, the three major bibliographic sources come from G. Matheron, Ch. Ronse, and H. Heijmans. The notion of an $\mathcal{R}$-open (closed) set recurrently appears in Matheron works, from 1969 until 1996. In [16], p.156-157, he builds a $\sigma$-algebra of regular closed sets and proves a series of characteristic properties; in [15] he associates a random set to every pair $\left(A^{\circ}, \bar{A}\right), A \in \mathcal{P}(E)$; in $[23]$ he interprets the regularization operator as a strong morphological filter, and gives a middle element between $\overline{(\bar{A})^{\circ}}$ and $\left(\overline{A^{\circ}}\right)^{\circ}$. For his part, Ch. Ronse shows in [21] that any complete boolean lattice is isomorphic to a lattice of regular open sets, and indicates as a watermark that the associated topology is Alexandrov. 
Besides, the set difference $\Delta=S \backslash B$ between $S$ and $B$ coincides with the difference between the boundaries $\operatorname{Fr}(B)$ and $\operatorname{Fr}(S)$ :

$$
\Delta=S \backslash B \Leftrightarrow \operatorname{Fr}(S)=\operatorname{Fr}(B) \backslash \Delta,
$$

and the frontier of $B$ contains that of $S$.

The open sets of a tessellation cannot admit lacunae or fissures of empty interior, which makes the tessellation impossible. Thus the convenient class to consider are that of the $\mathcal{R}$-open sets. Indeed,

Theorem 2. All classes $B_{i}$ of any tessellation $\tau=\left\{B_{i}, i \in I\right\}$ are necessarily $\mathcal{R}$-open.

Proof. We prove the theorem in the negative, by showing that if $B_{i} \neq\left(\overline{B_{i}}\right)^{\circ}=S_{i}$, then the $B_{i}$ do not generate a tessellation. We firstly observe that the $B_{i}$ are disjoint iff the corresponding $\mathcal{R}$-open sets $S_{i}$ are disjoint. We have

$$
B_{i} \cap B_{j}=\emptyset \Leftrightarrow B_{i} \cap \bar{B}_{j}=\emptyset \Rightarrow B_{i} \cap\left(\overline{B_{j}}\right)^{\circ}=\emptyset=B_{i} \cap S_{j} \Rightarrow S_{i} \cap S_{j}=\emptyset
$$

as well as the inverse implication, since $S_{i} \supseteq B_{i}, \forall i$. Besides, we have, from Rel. (5), $\cup\left\{\bar{B}_{i} \cap\right.$ $\left.\bar{B}_{j}, i, j \in, i \neq j\right\}=\cup\left\{\bar{S}_{i} \cap \bar{S}_{j}, i, j \in, i \neq j\right\}$ so that the $S_{i}$ form a tessellation of $E$. If there exists one $B_{i}$ at least which is strictly included in $S_{i}$ then $z \in S_{i} \backslash B_{i}$ does not belong to any $B_{i}$ or to any boundary $\bar{B}_{i} \cap \bar{B}_{j}$ since $S_{i}$ is open. Therefore the $B_{i}$ do not cover set $E$, which achieves the proof.

All in all, just as a partition of $E$ is a family in $\mathcal{P}(E)$ whose each element is the complement of the union of all the others, a tessellation of $E$ is a family in $\mathcal{R}(E)$ whose each element is the complement of the supremum of all the others, both complement and supremum being taken in $\mathcal{R}$.

The algebraic closing $B \rightarrow S=(\bar{B})^{\circ}$ fills up the fine fissure, isthmuses and the point lacunae of $B$. However, some separations, too narrow, may not be filled up. Take for example, $B$ composed of two disjoint open squares of $\mathbb{R}^{2}$ whose adherence share one vertex only, then the adherence $\overline{B_{1} \cup B_{2}}$ joins the two squares, but the interior $\left(\overline{B_{1} \cup B_{2}}\right)^{\circ}$ separates them again.

In the literature, an example of tessellation is given by the clefts in the perfect fusion graphs of J. Cousty and G. Bertrand [2]. Theorem 2 reminds us of Jordan's one, though it is true in any topological space, and it does not focus on the frontiers, but on the classes. In $\mathbb{R}^{2}$ every Jordan curve induces a tessellation [9], but a tessellation into two open classes, even connected, can have a frontier which is not a Jordan curve. Fig. 4b depicts a digital contour which separates $\mathcal{R}$-open sets and which is thick. Note also that connectivity is not involved in the above theorem, whereas it is essential in Jordan's one.

\subsection{Structure of the tessellations}

We now introduce a minimal tessellation $\tau_{0}$ of the space $E$. The classes $\left\{s_{i}\right\}$ of $\tau_{0}$ are called "the leaves", and are supposed in locally finite number. These leaves are indivisible $\mathcal{R}$-open sets, i.e. each class of a larger tessellation contains one leave at least and is disjoint from those that it does not contain. The family $\mathcal{T}$ of all tessellations of $E$ is obviously ordered by the following relation :

$$
\tau \leq \tau^{\prime} \quad \Leftrightarrow \quad S(x) \subseteq S^{\prime}(x) \quad x \in \tau_{0} \tau, \tau^{\prime} \in \mathcal{T}
$$

where $S(x)$ (resp. $S^{\prime}(x)$ ) is the class of $\tau$ (resp. $\tau^{\prime}$ ) at point $x$. This ordering provides $\mathcal{T}$ with the structure of a complete lattice. More precisely, consider a set $P$ of labels and the family $\left\{\tau_{p}, p \in P\right\}$ :

Proposition 1. The set $\mathcal{T}$ of all tessellations $\tau \geq \tau_{0}$ of $E$ forms a complete lattice for the ordering of Rel.(7), with universal elements $\tau_{0}$ and $E$. The infimum of family $\left\{\tau_{p}, p \in P, \tau_{p} \geq \tau_{0}\right\}$ is the tessellation whose class at point $x$ is the infimum, in $\mathcal{R}$, of the classes of the $\tau_{p}$ at point $x$, and the supremum is the smallest tessellation whose classes are suprema of the classes of the $\tau_{p}$ in $\mathcal{R}$. 
Proof. Let $x$ and $y$ be two classes of $\tau_{0}$, and $S_{p}(x)$ (resp. $S_{p}(y)$ ) be the class of $\tau_{p}$ at point $x$ (resp. $y$ ). We have to prove that $\left\{\left[\cap S_{p}(x)\right]^{\circ}, x \in \tau_{0}\right\}$ is a tessellation. If so, then this tessellation will be the greatest lower bound of the $\tau_{p}$, since on cannot find at point $x$ an $\mathcal{R}$-open class greater than $\left[\cap S_{p}(x)\right]^{\circ}$ and which is included in all $S_{p}(x)$.

The point $y \in S_{p}(x)$ iff $x \in S_{p}(y)$; therefore, if $y \in\left[\cap S_{p}(x)\right]$ then $\left[\cap S_{p}(x)\right]=\left[\cap S_{p}(y)\right]$ and the two $\mathcal{R}$-infima $\left[\cap S_{p}(x)\right]^{\circ}$ and $\left[\cap S_{p}(y)\right]^{\circ}$ are equal. If for some $p$ the two sets $S_{p}(x)$ and $S_{p}(y)$ are disjoint, then a fortiori the $\mathcal{R}$-infima $\left[\cap S_{p}(x)\right]^{\circ}$ and $\left[\cap S_{p}(y)\right]^{\circ}$ are also disjoint. Finally, as $\cup\left\{x, x \in \tau_{0}\right\} \subseteq \cup\left\{\left[\cap S_{p}(x)\right]^{\circ}, x \in \tau_{0}\right\}$, and as $\cup\left\{x, x \in \tau_{0}\right\}=E$, the family $\left\{\left[\cap S_{p}(x)\right]^{\circ}, x \in \tau_{0}\right\}$ turns out to be a tessellation of $E$, and $\mathcal{T}$ is a complete inf-semi Lattice. But in addition $\mathcal{T}$ admits a greatest element, namely $E$ itself, so that $\mathcal{T}$ is a complete lattice, which achieves the proof.

\subsection{Hierarchies of tessellations}

The tessellations met in image processing often form hierarchies, i.e. totally ordered closed sequences starting from the leaves and ending at $E$ itself, considered as an $R$-open class.

One cannot merge the classes of a tessellation $\tau$ of the hierarchy by performing a simple union of their leaves, since the frontiers between adjacent leaves (i.e. with non-empty intersection of the adherences) would neither belong to classes, nor to the background net $N$ which separates the classes. We must find out another law of composition. Consider a partition the totality of the leaves into $J$ sub-sets $B_{1}, . . B_{j}, . . B_{J}$, then

Proposition 2. The unique tessellation whose each class includes exactly one $B_{j}, 1 \leq j \leq J$ has the $\mathcal{R}$-open sets $S_{j}=\left(\overline{B_{j}}\right)^{\circ}, 1 \leq j \leq J$ as classes.

Proof. Theorem 2 shows that we must replace each $B_{j}$ with its $\mathcal{R}$-open version $S_{j}$ for finding again a tessellation. Moreover, there is no tessellation $\left\{S_{j}^{\prime}\right\}$ which keeps disjoint the family $\left\{S_{j}\right\}$ and such that $S_{j}^{\prime} \supset S_{j} \forall j$. Such a strict inclusion would mean that one could find an open set containing $x \in \operatorname{Fr}\left(S_{j}\right) \cap S_{j}^{\prime}$, such that $S_{j}^{\prime}$ is not disjoint from $S_{i}^{\prime}$, which is impossible.

The supremum and the infimum of a family of classes are those involved in the lattice $\mathcal{R}$ of the $\mathcal{R}$-open sets. Consequently, in a hierarchy of tessellations, every point of the net $N$ is absorbed by a class, sooner or later.

\section{Connected classes and saliency function}

Connectivity does not intervene in the paradigm of the hierarchies of tessellations. However the leaves are often connected, and the question of preserving the connectivity of the classes arises. Now this requirement is not always possible, neither in Euclidean topologies, nor in digital ones. Even when the leaves $\left\{s_{i}\right\}$ are connected, the $\mathcal{R}$-open sets $S_{j}=\left(\overline{B_{j}}\right)^{\circ}$ of Prop. 2 may be not connected. One can also think of the example of the two open squares of $\mathbb{R}^{2}$ whose adherences have only one point in common. They are connected, regular, their adherence is connected, but their union, though regular, is again not connected.

To overcome this issue, we have to specify the nature of topological space $E$, which is for now supposed to be locally arcwise connected, i.e. where the arcwise-connected components of an open set are open. Furthermore, we say that a pair of two regular sets $S_{i}$ and $S_{j}$ of $E$ is strongly connected when the sets are adjacent (i.e. the intersection of their adherences is not empty) and when one can find a point $x$ in their common frontier which is included in a small neighborhood $\delta(x)$ which itself is included in the union of their adherences,i.e.

$$
S_{i} \cap S_{j}=\emptyset \text { and } \exists x \in \overline{S_{i}} \cap \bar{S}_{j} \mid x \in \delta(x) \subseteq \overline{S_{i}} \cup \overline{S_{j}} .
$$

Hierarchies of tessellations require this strong adjacency to transmit the connectivity from the leaves to their clusters into classes:

Theorem 3. let $E$ be a locally arcwise connected set, and $\tau_{0}$ be a tessellation of $E$ into $\mathcal{R}$-open connected leaves $\left\{s_{i}, i \in I\right\}$ where every adjacent pair is strongly adjacency (8). The $\mathcal{R}$-supremum of any finite sequence $\left\{s_{k}, 1 \leq k \leq n\right\}$ of leaves is then connected. 


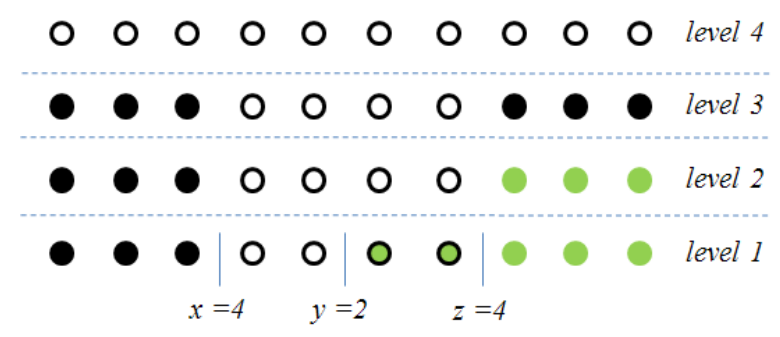

Fig. 2. The space $E$ is the 10 points of $\mathbb{Z}$. The saliency function $\{x, y, z\}$ does not detect the non connected class (in black) which appears at level 3 of this hierarchy.

Proof. Let $S=\vee\left\{s_{k}, 1 \leq k \leq n\right\}$ be the $\mathcal{R}$-supremum of the family. From Prop. $2, S$ is the union of the $\left\{s_{k}\right\}$ and of the boundaries between pairs $\left\{s_{k}, s_{l}\right\}$. Consider two strongly adjacent leaves $\left\{s_{k}, s_{l}\right\}$. By strong adjacency, there exists a point $x$ of the boundary $\overline{s_{k}} \cap \bar{s}_{l}$ which has a neighborhood $\delta(x) \subseteq \overline{s_{k}} \cup \bar{s}_{l}$. As $E$ is locally arcwise connected, there exists also a connected neighborhood $\delta^{\prime} \subseteq \delta(x)$ which thus contains points of $s_{k}$ and of $s_{l}$. Therefore the union $s_{k} \cup s_{l} \cup \operatorname{Fr}\left(s_{k}, s_{l}\right)$ is arcwise connected, and so is $S$, which achieves the proof.

In particular, the usual spaces $\mathbb{R}^{n}$ and $\mathbb{Z}^{n}$ are locally arcwise connected. Note that a same leave may appear several times in the sequence $\left\{s_{p}, 1 \leq p \leq n\right\}$. When a hierarchy $H$ with $n$ levels $\tau_{p}, 1 \leq p \leq n$ of tessellations satisfies the conditions of Theorem 3 then one can allocate a numerical value to each point of the net $N_{0}$ of the frontiers between the leaves of $H$. This function on $N_{0}, s$ say, indicates the level when the frontier element adjacent between two classes vanishes, and the classes merge.

This is nothing but the classical saliency function [19], here generalized, and without assuming that the boundaries are Jordan curves (in the case of $\mathbb{R}^{2}$ ). When the conditions of Theorem 3 are not satisfied, as in Fig. 1a, the saliency function may be undefined at some crucial points. Finally, when the classes are possibly not connected, the saliency function may no longer summarizes exhaustively the hierarchy, as shown in Fig. 2.

\section{$5 \quad$ Tessellations of $\mathbb{Z}^{n}$ and Khalimsky spaces}

The notion of a tessellation rests on $\mathcal{R}$-open sets, and to apply it to $\mathbb{Z}^{n}$, we first need to provide a topology to this space.

\subsection{Reminder on Khalimsky topology}

E. Khalimsky topology clearly shows the analogies between the tessellations of $\mathbb{R}^{n}$ and those of $\mathbb{Z}^{n}$. It was published in Russian in the sixties, but it is better known by more recent papers in English [8]. It is just sketched here, but the reader will find a more detailed presentation in papers by E. Melin [18], L. Mazo et Al. [17], and the lecture notes of Ch. Kiselman [11].

In 1937, P.S. Alexandrov introduced topological spaces $E$ with smallest neighborhood (in short sn-topology), where it is assumed that the class of the open sets is closed under intersection [20]. Khalimsky topology belongs to this category. In $\mathbb{R}$, it associates the open interval $] m-\frac{1}{2}, m^{\prime}+\frac{1}{2}[$ with every pair $m \leq m^{\prime}$ of odd integers, and the closed interval $\left[n-\frac{1}{2}, n^{\prime}+\frac{1}{2}\right]$ with every pair $n \leq n^{\prime}$ of even integers. The unions of open (resp. closed) intervals generate a non separated sntopology. When $m=m^{\prime}$ and $n=n^{\prime}$ then $\mathbb{R}$ is partitioned into unit intervals, and is thus connected. The product topology of $n$ Khalimsky lines generates the topology in $\mathbb{R}^{n}$. When all coordinates of their centers are odd (resp. even), then the $n$-cubes are open (resp.closed), and the others $n$-cubes are said to be mixed.

In $\mathbb{R}^{2}$ the Kovalevsky cells provide an equivalent approach, which allow us to display these topologies [12]. This structure is akin to the simplicial complexes [1], to fusion grids [2] and to the planar graphs. 
According to Theorem 2, in a Khalimsky space the tessellations are $\mathcal{R}$-open sets, which simplifies the basic elements. In $\mathbb{Z}^{2}$ for example, the isolated points and the pending edges are removed. The background net $N$ is made of loops contouring the classes and where open edges and points alternate (see Fig. 3).
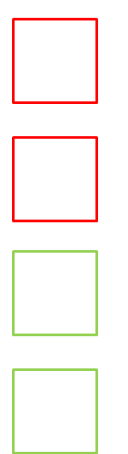
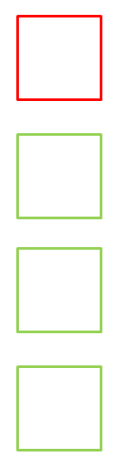

$a$
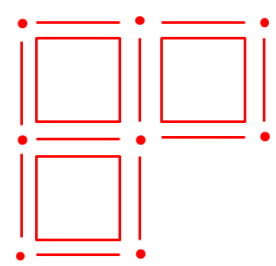
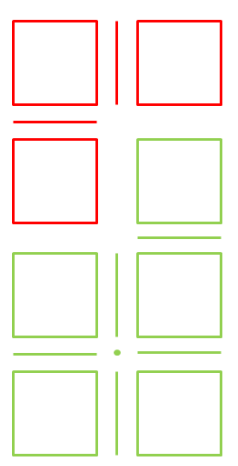
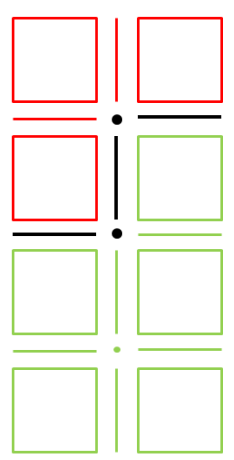

d

Fig. 3. Simple resolution: a) two classes of a partition of $\mathbb{Z}^{2}$. Double resolution: b) Khaminsky closure of the first class of Fig.a; c) regular open versions of the two classes; d) corresponding tessellation (in black, the net between classes).

\subsection{Khalimsky digital tessellations}

The use of Khalimsky topology in digital image processing is classical $[17,18]$. In this section we indicate its links with the $\mathcal{R}$-open sets and tessellations, and we show that this topology does not solve the question of the diagonal configurations.

Khalimsky topology on $\mathbb{Z}$ is obtained by identifying each unit interval of $\mathbb{R}$ with the corresponding integer. The open intervals are obtained in the same manner from $] m-\frac{1}{2}, m^{\prime}+\frac{1}{2}$ [ where $m$ and $m^{\prime}$ are odd and $m \leq m^{\prime}$. In this topology $\mathbb{Z}$ is connected. The extension to $\mathbb{Z}^{n}$ is made as previously with $\mathbb{R}^{n}$.

Interpret the points of a set $X \subseteq \mathbb{Z}^{2}$ as points of odd coordinates in a Khalimsky plane $\mathbb{K}^{2}$ which contains twice more points by line and twice more lines [3,4,9]. In Kovalevsky representation, the initial points of $X \subseteq \mathbb{Z}^{2}$ become squares and the additional points become segments and points, as depicted in Fig. 3b. The adherence $X \rightarrow \bar{X}$ is the union of the adherences of its basic elements, and the interior $\bar{X} \rightarrow(\bar{X})^{\circ}$ is the complement of the adherence of the complement set. Fig. 3b depicts the step $X \rightarrow \bar{X}$ for one class of $X$, and Fig. 3c $\bar{X} \rightarrow(\bar{X})^{\circ}$ depicts the construction of the regularized version $(\bar{X})^{\circ}$. The two classes of tessellation $(\bar{X})^{\circ}$ are separated by the net in bold of Fig. 3d. In the double resolution plane $\mathbb{K}^{2}$, all points of the background have odd coordinates. This example illustrates the following property:

Proposition 3. Let $\mathbb{K}^{n}$ be the Khalimsky space of $n$ dimensions and $\mathbb{Z}^{n}$ be the sub-space formed by the points of $\mathbb{K}^{n}$ whose all coordinates are odd. Every partition $\pi$ of $\mathbb{Z}^{n}$ induces in $\mathbb{K}^{n}$ a unique tessellation $\tau$ whose each class contains one class of $\pi$, and the correspondence between $\pi$ and $\tau$ is biunivocal.

Proof. When $\mathbb{Z}^{n}$ is embedded in $\mathbb{K}^{n}$, each class $B_{i}$ of $\pi$ becomes open, since all its coordinates are odd. Replace all $B_{i}$ by their regularized versions $S_{i}=\left(\overline{B_{i}}\right)^{\circ}$, and let $z$ be a point of $\mathbb{K}^{n}$ which does not belongs to any class, i.e. $z \in\left[\cup S_{i}\right]^{c}$. Point $z$, which has one even coordinate at least, is the center of a unit cube which meets two classes $S_{i}$ at least, which amounts to say that the $\overline{S_{i}}$ cover the space. Thus the family $\left\{S_{i}\right\}$ forms a tessellation of $\mathbb{K}^{n}$. Conversely, let $\tau$ be a tessellation of $\mathbb{K}^{n}$. Those points whose all coordinates are odd lie necessarily in some classes, as they are open, therefore the inverse passage to $\mathbb{Z}^{n}$ forms a partition. 
This proposition theoretically justifies the classical rule of the double sampling $[3,4,10]$. The fine mesh displays the net of the contours, which are topologically closed in $\mathbb{R}^{n}$ as in $\mathbb{K}^{n}$, and which envelop the connected open sets. The odd points of $\mathbb{K}^{n}$, i.e. the unit open cubes play exactly the same role as the leaves in a hierarchy. But the topology of $\mathbb{K}^{n}$ does not remove the ambiguity of the diagonals, as shown in Fig.1a. We must introduce another topology.

\section{Tessellations of $\mathbb{Z}^{2}$ and $\mathbb{Z}^{3}$ by Voronoi polyhedra}

Let $X \subseteq \mathbb{R}^{n}$ be a locally finite set of centers. One can always associate with each center $x \in X$ the so-called Voronoi polyhedron $Q(x)$ of all points $y \in \mathbb{R}^{n}$ closer to $x$ than to any other center. $Q(x)$ is convex and open, hence regular, so that the set $\{Q(x), x \in X\}$ of all Voronoi polyhedra generates a tessellation of $\mathbb{R}^{n}$. In particular in $\mathbb{R}$, when the centers are the points $m$ of odd integer abscissae $m$, the corresponding Voronoi polyhedron is $] m-1, m+1[$. We find again Khalimsky topology.

Let $\mathcal{H}^{3}$ represent the truncated octahedron. Let us come back to $\mathbb{R}^{n}$ and impose the following two conditions to the Voronoi polyhedra:

1. they must be identical, up to a translation (i.e. regular grid);

2. the adherences of two adjacent polyhedra always have a common face of $n$ - 1 dimension.

Concerning the first constraint, the mineralogist E.S. Fedorov proved that there are only two solutions in $\mathbb{R}^{2}$, the square and the hexagon, and five in $\mathbb{R}^{3}$, the cube, the hexagonal prism, the truncated octahedron, and the two elongated and rhombic dodecahedra [5].

The second condition imposes strong adjacencies, thus preserves connectivity, according to Theorem 3. This condition reduces Fedorov possibilities to the only hexagon in the plane, and only truncated octahedron in the space. In the 2-D case the centers describe the triangular grid, in the 3-D case the centered cubic grid [24] (see Fig. 5a). If we are not interested in preserving connectivity, this second condition becomes cumbersome.

\subsection{Hexagonal tessellation of $\mathbb{Z}^{2}$}

In spite of its advantages the hexagonal grid is not often used (though it recently re-appeared about the simplicial complexes for digital watersheds [3]). Consider in $\mathbb{R}^{2}$ three axes of coordinates at $120^{\circ}$, and the origin $O$. Take for centers all points of the plane whose coordinates are odd on each of the three axes. The associated Voronoi polygons are open hexagons. The other open sets are obtained by unions of these hexagons plus the edges adjacent between them. They result in a sn-topology where the triple points are closed. In Fig. 4b the previous squares and hexagons are replaced by the unique symbol of big discs, which indicate the pixels of $X \subseteq \mathbb{Z}^{2}$, and the small points are added to give the $(\bar{X})^{\circ}$. The asterisks indicate the net of the frontiers of the tessellation in the double resolution. By comparison with Khalimsky tessellation in square grid (Fig. 4a) two major differences appear. On the one hand, the frontiers are no longer Jordan arcs (clusters of pixels in the hexagonal grid), and on the other hand the ambiguous diagonal were removed by suppression of the quadruples points. If Fig. 4b is interpreted as the leaves of a hierarchy, a unique value is allocated to each crossing point, namely that of the level when North-East and South-West classes merge.

\subsection{Tessellation of $\mathbb{Z}^{3}$ by truncated octahedra}

The Voronoi polyhedra of the centered cubic grid are the truncated octahedra depicted in Fig. 5. They partition $\mathbb{R}^{3}$ in open polyhedra, square and hexagonal faces, triple edges and quadruple vertices. These elements generate a sn-topology. The regularization fills up the internal 1-D or 2-D fissures of zero thickness, and the background net is a connected union of faces and edges which completely envelops the classes.

In the digital version, the double resolution rule for inducing a tessellation is indicated in Fig. 5. One starts from three horizontal planes of the cubic grid containing the vertices of the unit cube $\left(\mathrm{n}^{\circ} 1\right.$ and 5 in the Fig.), and the center $\left(\mathrm{n}^{\circ} 3\right)$. The planes $\mathrm{n}^{\circ} 2$ and 4 are added for generating a 

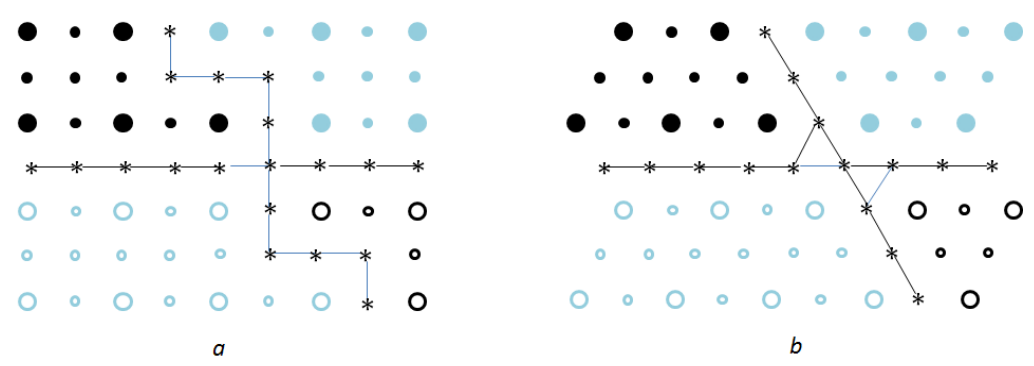

Fig. 4. Square versus hexagonal tessellation. The big discs are the elementary open sets; the unions of big and small discs form the regular open sets the small discs represent the segments and the points (square grid); and the contours with segments and asterisks delineate the background net. In the hexagonal case the quadruple point vanishes (thus its inherent ambiguity), but the background is no longer threadlike (small triangles).

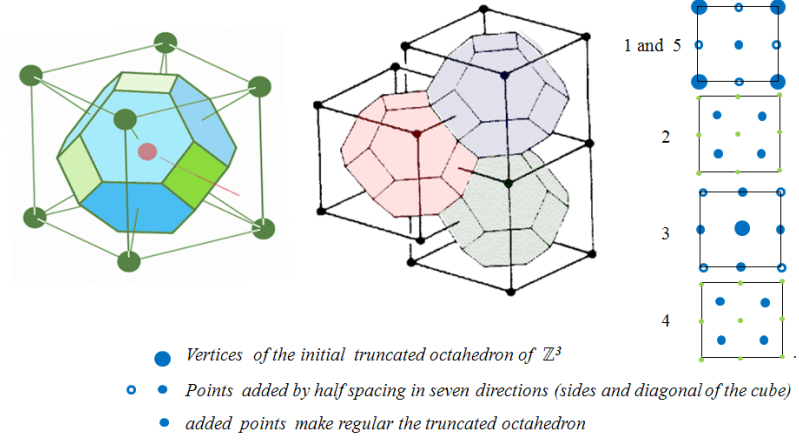

Fig. 5. Left: Truncated octahedron and centered cubic grid. Center: Partition the space. Right: Top-down sections of the truncated octahedron. The big points are the open truncated octahedric cells of $\mathbb{Z}^{3}$, the small points and the rings are the points added to make $\mathbb{H}^{3}$. The regularization is obtained by union of big and small voxels.

centered cubic grid twice finer. In the three directions of the cube and the four ones of the main diagonals alternate points of $\mathbb{Z}^{3}$ with those added for forming the double resolution space.

Again the tessellation reduces the cells to the two types of the (open) truncated octahedra, and the (closed) square or hexagonal faces, i.e. something that can be described in terms of graphs. If the first elements are displayed by points and the second by asterisks, like in Fig. 4 in 2-D, and if we connect the asterisks which share an edge, then each class turns out to be a cluster of points completely surrounded by a net of asterisks. In practice, the centered cubic grid can easily be emulated by shifting horizontally the even planes by the vector $(1,1,0)$.

\section{Conclusion}

In this paper, we characterized the partitions whose interiors and frontiers of the classes are jointly represented. They are the tessellations, consist of $\mathcal{R}$-open sets. No conditions like to be Jordan curves (in 2D) hold on the frontiers, which can have a certain thickness. The theory applies to both Euclidean and digital spaces, which gives more soundness to the numerical techniques. It was shown in detail how the notions of tessellation, Alexandrov topology, and double resolution interact. In digitizing a partition we make use of the double resolution.

For $\mathbb{Z}^{3}$ we proposed a variant of Khalimsky topology which suppresses the ambiguities of the diagonal configurations, it is based on the truncated octahedra, that is the regular vornoi polyhedra of the centerd-cubic grid.

Finally, it would be interesting to consider other alegraic closing operations, since they would also treat the classes and their frontiers in a unique representation. 
Acknowledgment : The authors acknowledge G. Bertrand J. Cousty and T. Geraud for their useful comments, and the two reviewers for their pertinent remarks.

\section{References}

1. Bertrand, G.: Completions and simplicial complexes. In: Debled-Rennesson, I., Domenjoud, E., Kerautret, B., Even, P. (eds.) DGCI 2011, Nancy, France, April 6-8, 2011. Proceedings. LNCS, vol. 6607, pp. 129-140. Springer (2011)

2. Cousty, J., Bertrand, G.: Uniqueness of the perfect fusion grid on $z^{d}$. JMIV 34(3), 291-306 (2009)

3. Cousty, J., Bertrand, G., Couprie, M., Najman, L.: Collapses and watersheds in pseudomanifolds of arbitrary dimension. JMIV 50(3), 261-285 (2014)

4. Cousty, J., Najman, L., Serra, J.: Some morphological operators in graph spaces. In: Wilkinson, M.H.F., Roerdink, J.B.T.M. (eds.) ISMM 2009, Groningen, The Netherlands, August 24-27, 2009 Proceedings. LNCS, vol. 5720, pp. 149-160. Springer (2009)

5. Fedorov, E.S.: A Course in Crystallography (In Russian). R. K. Rikker, Saint-Petersburg (1901)

6. G., M.: Les treillis compacts. Tech. rep., Ecole des Mines, Paris (1996)

7. Heijmans, M.: Morphological Image Operators. Boston: Academic Press (1994)

8. Khalimsky, E.: Topological structures in computer science. International Journal of Stochastic Analysis 1(1), 25-40 (1987)

9. Kiran, B.R., Serra, J.: Fusion of ground truths and hierarchies of segmentations. Pattern Recogn. Lett. 47, 63-71 (oct 2014)

10. Kiran, B.R.: Energetic-Lattice based optimization. Ph.D. thesis, Université Paris-Est (2014)

11. Kiselman, C.O.: Digital geometry and mathematical morphology. Lecture Notes, Uppsala University, Departement of Mathematics (2002)

12. Kovalevsky, V.A.: Finite topology as applied to image analysis. Computer vision, graphics, and image processing 46(2), 141-161 (1989)

13. Kronheimer, E.: The topology of digital images. Topology and its Applications 46(3), 279-303 (1992)

14. Latecki, L., Eckhardt, U., Rosenfeld, A.: Well-composed sets. Computer Vision and Image Understanding 61(1), 70-83 (1995)

15. Matheron, G.: Random Sets and Integral Geometry. Wiley (1975)

16. Matheron, G.: Eléments pour une théorie des milieux poreux (1967)

17. Mazo, L., Passat, N., Couprie, M., Ronse, C.: Paths, homotopy and reduction in digital images. Acta applicandae mathematicae 113(2), 167-193 (2011)

18. Melin, E.: Digital surfaces and boundaries in khalimsky spaces. JMIV 28(2), 169-177 (2007)

19. Najman, L., Schmitt, M.: Geodesic saliency of watershed contours and hierarchical segmentation. PAMI, IEEE Transactions on 18(12), 1163-1173 (1996)

20. P., A.: Diskrete raüme rec. math. [mat. sbornik] (1937)

21. Ronse, C.: Regular open or closed sets. Working Document WD59, Philips Research Lab., Brussels (1990)

22. Rosenfeld, A.: Digital topology. American Mathematical Monthly pp. 621-630 (1979)

23. Serra, J.: Image Analysis and Mathematical Morphology, Vol. 2: Theoretical Advances. London: Academic Press (1988)

24. Serra, J.: Cube, cube-octahedron or rhombododecahedron as bases for 3-d shape descriptions. Advances in Visual Form Analysis, C. Arcelli and Al.(eds.) pp. 502-519 (1997)

25. Serra, J.: Image Analysis and Mathematical Morphology. Academic Press, Inc., Orlando, FL, USA $(1983)$ 